\title{
Monitoramento do binônimo tempo e temperatura de refeições transportadas para o jantar de instituições de privação de liberdade
}

\author{
Monitoring the dual time and temperature of meals transported to dinner in deprivation of liberty \\ institutions
}

Seguimiento de la doble hora y temperatura de las comidas transportadas a la cena en instituciones de privación de libertad

Fábio Jobson Ferreira Campos ORCID: https://orcid.org/0000-0001-5768-8924 ISM Gomes de Mattos EIRELI, Brasil E-mail: fabio.jobson@ism.ind.br

Denise Regina de Oliveira Brasil ORCID: https://orcid.org/0000-0002-5447-7360 ISM Gomes de Mattos EIRELI, Brasil E-mail: denise@ism.ind.br

Wilma Stella Giffoni Vieira

ORCID: https://orcid.org/0000-0002-9787-1157 ISM Gomes de Mattos EIRELI, Brasil E-mail: wilma.giffoni@ism.ind.br

Francisco Batista Moura Júnior ORCID: https://orcid.org/0000-0003-4583-8520 ISM Gomes de Mattos EIRELI, Brasil E-mail: franciscomourajr@gmail.com

Homero de Sousa Lima Junior ORCID: https://orcid.org/0000-0002-9373-3777 ISM Gomes de Mattos EIRELI, Brasil

E-mail: homero_jr@outlook.com

Renata Carmo de Assis

ORCID: https://orcid.org/0000-0003-2258-3844 Universidade Estadual do Ceará, Brasil E-mail: renata.carmo@uece.br

Amauri Barbosa da Silva Junior ORCID: https://orcid.org/0000-0002-1647-2331 Universidade Estadual do Ceará, Brasil E-mail: amauribdsj@gmail.com

Clarice Maria Araújo Chagas Vergara ORCID: https://orcid.org/0000-0003-1709-9951 Universidade Estadual do Ceará, Brasil E-mail: clarice.araujo@uece.br

\begin{abstract}
Resumo
Unidades de Alimentação e Nutrição (UAN) são locais voltados para a preparação e fornecimento de refeições equilibradas em nutrientes, seguindo o perfil da clientela. Os tratados internacionais de direitos humanos incluem duas dimensões que são indivisíveis do Direito Humano à Alimentação Adequada (DHAD), sendo os direitos de não ter fome e de consumir uma alimentação adequada, contudo, sabe-se que em grande parte dos presídios a alimentação não é bem aceita. Os alimentos por serem considerados veículos de agentes tóxicos, devem ser submetidos a práticas que evitam a propagação de doenças, para isso, existem medidas como a verificação do binômio tempo x temperatura que ajudam a evitar a proliferação microbiológica. O presente trabalho teve como objetivo analisar o binômio tempo x temperatura de refeições transportadas para o jantar de instituições de privação de liberdade na Região Metropolitana de Fortaleza no estado do Ceará. A amostra foi composta por 50 coletas de temperatura por dia, durante 8 dias, não sequenciais, totalizando 400 amostras. As refeições eram constituídas por uma opção proteica, um acompanhamento e uma guarnição, sendo que em 4 dos 8 dias, foram servidas uma opção de salada cozida produzida para o jantar. Os resultados obtidos indicaram que apenas 7,3\% das 400 amostras encontraram-se abaixo de $60^{\circ} \mathrm{C}$ na hora do recebimento, contudo, durante o envase das quentinhas $100 \%$ apresentaram temperaturas acima do recomendado pela legislação.
\end{abstract}

Palavras-chave: Controle de qualidade; Inocuidade dos alimentos; Segurança alimentar; Temperatura. 


\begin{abstract}
Food and Nutrition Units (UAN) are places dedicated to the preparation and supply of meals balanced in nutrients, following the profile of the clientele. International human rights treaties include two dimensions that are indivisible from the Human Right to Adequate Food (DHAD), being the rights not to be hungry and to consume adequate food. is well accepted. As foods are considered vehicles of toxic agents, they must be subjected to practices that prevent the spread of diseases. For this, there are measures such as checking the binomial time $\mathrm{x}$ temperature that help to prevent microbiological proliferation. This study aimed to analyze the time $\mathrm{x}$ temperature binomial of meals transported to dinner at institutions of deprivation of liberty in the Metropolitan Region of Fortaleza in the state of Ceará. The sample consisted of 50 temperature collections per day, for 8 days, non-sequentially, totaling 400 samples. The meals consisted of a protein option, a side and a garnish, and in 4 of the 8 days, a cooked salad option produced for dinner was served. The results obtained indicated that only $7.3 \%$ of the 400 samples were found to be below $60^{\circ} \mathrm{C}$ at the time of receipt, however, during the filling of the hot dishes, $100 \%$ had temperatures above those recommended by law.
\end{abstract}

Keywords: Quality control; Food safety; Food security; Temperature.

\title{
Resumen
}

Las Unidades de Alimentación y Nutrición (UAN) son lugares dedicados a la preparación y suministro de comidas balanceadas en nutrientes, siguiendo el perfil de la clientela. Los tratados internacionales de derechos humanos incluyen dos dimensiones que son indivisibles del Derecho Humano a una Alimentación Adecuada (DHAD), que son los derechos a no morir de hambre y a consumir alimentos adecuados. es bien aceptado. Al ser considerados vehículos de agentes tóxicos, los alimentos deben someterse a prácticas que eviten la propagación de enfermedades. Para ello, existen medidas como comprobar el binomio tiempo x temperatura que ayudan a prevenir la proliferación microbiológica. Este estudio tuvo como objetivo analizar el binomio tiempo x temperatura de las comidas transportadas a la cena en instituciones de privación de libertad de la Región Metropolitana de Fortaleza en el estado de Ceará. La muestra consistió en 50 recolecciones de temperatura por día, durante 8 días, de forma no secuencial, totalizando 400 muestras. Las comidas consistieron en una opción de proteínas, un acompañamiento y una guarnición, y en 4 de los 8 días se sirvió una opción de ensalada cocida elaborada para la cena. Los resultados obtenidos indicaron que solo el $7.3 \%$ de las 400 muestras estuvieron por debajo de $60^{\circ} \mathrm{C}$ al recibirlas, sin embargo, durante el llenado de platos calientes, el $100 \%$ tuvo temperaturas por encima de las recomendadas por ley.

Palabras clave: Control de calidad; Seguridad alimenticia; Seguridad alimentaria; Temperatura.

\section{Introdução}

Unidades de Alimentação e Nutrição (UAN) são espaços voltados para preparação e fornecimento de refeições equilibradas em nutrientes, segundo o perfil da clientela. O objetivo primário de uma UAN é servir refeições saudáveis do ponto de vista nutricional e seguras do ponto de vista higiênico-sanitário, no sentido de manutenção e/ou recuperação da saúde do comensal, visando auxiliar no desenvolvimento de hábitos alimentares saudáveis (Ricarte et al., 2008).

Os tratados internacionais de direitos humanos incluem duas dimensões que são indivisíveis do Direito Humano à Alimentação Adequada (DHAD), sendo os direitos de não ter fome e de consumir uma alimentação adequada, estes direitos, segundo a OMS, têm influência direta na vida da população encarcerada, contudo, em grande parte dos presídios no país não é bem aceita, sendo umas das principais reclamações as condições higiênicas sanitárias (Audi et al., 2018; Brasil, 2009; Gatherer, 2007).

A produção de refeição do sistema prisional no Brasil se dava através de uma distribuição centralizada, apresentando deficiências em relação ao alto custo para a manutenção dos padrões higiênicos sanitários da alimentação da população carcerária, porém, com os passar dos anos, foi adotado outra forma de distribuição das refeições, sendo ela a descentralização. Com a descentralização da produção e distribuição dos alimentos deste grupo, a administração do sistema penitenciário pode obter alguns benefícios, tais como: implantação de um sistema moderno, evitando falhas no fornecimento pela transferência a terceiros da administração e confecção da alimentação, redução dos custos de manutenção de área física e reposição de equipamentos de cozinha e demais materiais e utensílios, além de um menor risco de contaminação e de Doenças Transmitidas por Alimentos (DTA's); proporcionam o fornecimento de dietas equilibradas, de acordo com os padrões nutricionais exigidos; despreocupação com a estocagem de gêneros, quantidade e prazos de validade, entre outros (Dariva, 2013; Paredes, 2005). 
Contudo, com a implantação da alimentação descentralizada, cujos alimentos são produzidos e, em seguida, transportados para o local de consumo e com o conhecimento dos fatores que favorecem ou inibem a presença de microrganismos presentes na água ou alimento contaminado que afetam o organismo humano, é imprescindível evitar riscos para ocorrência de DTA's (Kawasaki, 2007; Van Amson, 2006).

Os alimentos são considerados veículos para agentes tóxicos, podendo ser contaminados ao longo de todo o seu processo produtivo, por qualquer substância estranha que entre em contato com o mesmo tornando-o inadequado ao consumo, estes agentes tóxicos podem ser de natureza química, física ou microbiológica (Tondo \& Bartz, 2014).

Silva Júnior (2014) destaca os fatores que influenciam na proliferação de microrganismos patogênicos, sendo eles a preparação com excessiva antecipação, alimentos deixados à temperatura ambiente, alimentos esfriados em panelas grandes, inadequada conservação à quente, descongelamento inadequado e preparação de quantidades de alimentos excessivas. O aquecimento ou cocção insuficiente e o reaquecimento inadequado influenciam na sobrevivência desses agentes patógenos.

As Boas Práticas de Fabricação (BPFs) são um conjunto de práticas e procedimentos cujo obtivo é o correto manuseio e preparo do alimento com a finalidade de reduzir ao máximo os riscos de contaminação do mesmo (Chaves et al., 2006). Se feita de forma correta a implementação das BPFs são uma forma eficaz de melhorar o controle de qualidade dos alimentos, proporcionando maior segurança aos produtores e consumidores (Figueiredo, 2003). Uma das técnicas principais técnicas de boas práticas no preparo de alimentos é o binômio tempo x temperatura (Bozatski, 2011).

O monitoramento do binômio tempo x temperatura é algo indispensável ao longo da produção alimentícia, pois o mesmo ajuda a garantir a inocuidade do alimento, devendo ser observado como uma forma de prevenção de multiplicação microbiológica, visto que os mesmos possuem faixas de temperatura que propiciam seu desenvolvimento, sendo necessária uma aferição constante (Borges et al., 2016; Cardoso \& Tarzia, 2016; Guerra, 2016). Além deste fator, também deve-se levar em consideração as condições dos meios de transporte envolvidos nestes serviços, devendo ser rigidamente controlados, afim de evitar a proliferação bacteriana, bem como a deterioração dos alimentos distribuídos (Moura Júnior, 2018).

A investigação a respeito da qualidade dos alimentos prontos permite verificar o atendimento à legislação, alertando sobre a importância do papel dos estabelecimentos que produzem e distribuem alimentos e das autoridades sanitárias na garantia de segurança alimentar da população (Chouman, 2010).

Considerando a necessidade de estudos referentes ao controle térmico em alimentação transportada, o presente estudo tem como objetiva avaliar o binômio tempo e temperatura de refeições transportadas para o jantar de instituições de privação de liberdade na Região Metropolitana de Fortaleza no estado do Ceará.

\section{Metodologia}

Trata-se de um estudo descritivo realizado na sede de uma Unidade de Alimentação e Nutrição (UAN) que fornece alimentação do tipo transportada para a região metropolitana de Fortaleza, Ceará.

Foi realizado monitoramento de temperaturas de refeições prontas e acondicionadas em embalagens de alumínio (tipo "quentinhas") em duas instituições de privação de liberdade da região metropolitana de Fortaleza - CE. Nesta modalidade de serviço, as embalagens são acondicionadas em caixas térmicas tampadas e transportadas em veículos do tipo caminhão de carroceria fechada, isotérmico, com paredes lisas e impermeáveis em PVC e piso em inox.

Para escolha dos locais foi levado em consideração as distâncias e os tempos médios de transportes. A mais próxima unidade A - está localizada a 10 quilômetros $(\mathrm{km})$ com um tempo médio de transporte de 22 minutos e a mais distante - unidade B - está localizada a $35 \mathrm{~km}$ com um tempo médio de 47 minutos, partindo da unidade distribuidora até as respectivas unidades. 
A amostra foi composta por 50 coletas de temperatura por dia, durante 8 dias, não sequenciais, iniciando no dia 11 de março a 05 de abril do corrente ano, totalizando 400 amostras. As refeições eram constituídas por uma opção proteica, um acompanhamento e uma guarnição, sendo que em 4 dos 8 dias, fora servida uma opção de salada cozida produzida para o jantar.

As temperaturas das amostras foram monitoradas na unidade produtora por tecnólogos em alimentos e nutricionistas, nos processos de cocção, manutenção a quente (envase/espera) e manutenção no transporte (início) e registradas em registros específicos da NBR 15.635/2015 - COE (Controle Operacional Essencial), sendo, respectivamente, codificados em COE 2.1, COE 4 e COE 4.2. No recebimento na unidade prisional, as medições foram feitas utilizando-se um termômetro digital do tipo espeto, da marca Digital Pocket®, calibrado em 30 de julho de 2018 , com variação de temperatura entre $-50^{\circ} \mathrm{C}$ a $+200^{\circ} \mathrm{C}$, no centro geométrico das preparações e foram registradas em formulário próprio, desenvolvido especificamente para esta pesquisa. O horário da aferição foi medido através de um relógio comum. As temperaturas foram comparadas com a Portaria CVS 5 de 2013 e com a RDC n²16/2004.

Com o propósito e o cuidado de garantir o recebimento das quentinhas com uma temperatura que contribua para a garantia da qualidade microbiológica das refeições, foi realizado também a coleta das temperaturas de envase e se, por ventura, o alimento apresentasse temperatura inferior a $60^{\circ} \mathrm{C}$ no envase o alimento seria reaquecido para que permanecesse em temperatura favorável à manutenção.

O procedimento de utilização do termômetro seguiu as recomendações da ABERC (2015), que preconiza a higienização do termômetro com álcool $70 \%$ antes e após a medição de cada preparação.

A técnica de montagem foi fator determinante, onde finalizado o processo de cocção do alimento, o mesmo segue para a linha de montagem/envase, e a refeição montada, por sua vez, segue, sem interrupções, para o veículo de acordo com a ordem de saída na expedição.

\section{Resultados e Discussão}

Ao observar a Tabela 1 pode-se constatar que 7,3\% das quentinhas foram recebidas com temperaturas inferiores a $60^{\circ} \mathrm{C}$ e $92,7 \%$ para as recebidas igual ou superior a $60^{\circ} \mathrm{C}$. Isso representa que 371 amostras $(92,7 \%)$ estavam acima de $60^{\circ} \mathrm{C}$. Deste modo, 92,7\% das quentinhas chegaram com temperatura satisfatória ao destino final, o que contribui para a garantia microbiológica dos alimentos. Em contrapartida, 7,3\% estavam abaixo dos $60^{\circ} \mathrm{C}$, todavia vale salientar a margem de tolerância de 1 hora para consumo. Nas etiquetas de identificação da refeição há uma mensagem que alerta que o consumo deve ser imediato. Os dados obtidos divergem dos resultados encontrados por Abreu e Colaboradores (2012), que evidenciaram resultado inferior em suas análises. Eles realizaram o estudo seguindo o fluxo de duas linhas. A média encontrada foi de $88 \%$ das temperaturas abaixo de $60^{\circ} \mathrm{C}$ e apenas $12 \%$ entre $60^{\circ} \mathrm{C}$ e $65^{\circ} \mathrm{C}$.

Tabela 1: Representação numérica e percentual do quantitativo de amostras na etapa de recebimento segundo o critério de temperatura. Fortaleza, 2019.

\begin{tabular}{c|c|c|c|c|c|c|c|c|c}
\hline & $\begin{array}{c}\text { Abaixo } \\
\text { de } \\
6^{\circ} \mathbf{C}\end{array}$ & $\%$ & $\begin{array}{c}\text { Entre } \\
6^{\circ} \mathbf{e}\end{array}$ & $\begin{array}{c}\text { Entre } \\
\mathbf{6 5}^{\circ} \mathrm{C}\end{array}$ & $\begin{array}{c}\mathbf{6 5}^{\circ} \mathrm{e} \\
\mathbf{7 0}^{\circ} \mathrm{C}\end{array}$ & $\begin{array}{c}\text { Acima } \\
\text { de } \\
\mathbf{7 0}^{\circ} \mathrm{C}\end{array}$ & $\begin{array}{c}\text { TOTAL } \\
\text { AMOSTRAS }\end{array}$ \\
\hline $\mathbf{N}^{\circ}$ Amostras & 29 & $7,3 \%$ & 244 & $\mathbf{6 1 , 0 \%}$ & $\mathbf{8 2}$ & $\mathbf{2 0 , 5 \%}$ & $\mathbf{4 5}$ & $\mathbf{1 1 \%}$ & $\mathbf{4 0 0}$ \\
\hline
\end{tabular}

Fonte: Dados obtidos pelo pesquisador. 
Borges e colaboradores (2016), observaram que 100\% das preparações quentes de um Restaurante Universitário, encontravam-se dentro do preconizado pela legislação, já Santos e Bassi (2015), realizaram o estudo em oito unidades de alimentação na cidade de São Paulo, contudo, foram observados valores muito elevados de inadequação em relação a arroz/feijão e guarnições, apresentando $87,5 \%$ e $62,5 \%$ respectivamente, valores bem distintos aos encontrados no presente estudo.

Peixoto, Carneiro e Cardoso (2020), obtiveram valores distintos ao serem comparados as preparações em geral, onde $65,7 \%$ apresentaram temperaturas acima de $60^{\circ} \mathrm{C}$, porém ao analisar as preparações separadamente, observa-se que esta porcentagem é reduzida para $60 \%$ quando visto apenas guarnições, sendo esta a que obteve menor porcentagem de adequação no estudo, contudo, o autor saliente que este grupo foi o que se comportou de forma mais heterogênea, variando muito em relação aos diferentes tipos de guarnição que foram ofertados.

Tabela 2: Temperaturas $\left({ }^{\circ} \mathrm{C}\right)$ das preparações na etapa de manutenção a quente (envase). Fortaleza, 2019.

\begin{tabular}{l|c|c|c|c|c|c|c|c}
\hline \multicolumn{1}{c|}{ Dias } & $\mathbf{1}$ & $\mathbf{2}$ & $\mathbf{3}$ & $\mathbf{4}$ & $\mathbf{5}$ & $\mathbf{6}$ & $\mathbf{7}$ & $\mathbf{8}$ \\
\hline Acompanhamento & 90,0 & 88,0 & 76,0 & 66,0 & 66,0 & 85,0 & 73,0 & 91,0 \\
\hline Guarnição & 72,0 & 60,0 & 62,0 & 63,0 & 63,0 & 61,0 & 62,0 & 60,0 \\
\hline Opção Proteica & 75,0 & 87,0 & 92,0 & 85,0 & 75,0 & 92,0 & 64,0 & 92,0 \\
\hline Salada & 68,0 & NA & 64,0 & NA & 64 & NA & 69,0 & NA \\
\hline
\end{tabular}

Fonte: Dados obtidos pelo pesquisador.

Pode-se constatar que não houveram registros de temperaturas inferiores a $60^{\circ} \mathrm{C}$ na área de envase. A média das temperaturas de envase para o acompanhamento foi de $79,3^{\circ} \mathrm{C}$, para a guarnição foi de $62,8^{\circ} \mathrm{C}$, para opção proteica $82,7^{\circ} \mathrm{C}$ e para a salada de $66,2^{\circ} \mathrm{C}$.

Foi observado durante o processo que a preparação "baião de dois", durante a sua cocção, chega a uma temperatura média de $100^{\circ} \mathrm{C}$, chegando à linha de montagem, em recipientes produzidos em aço inox que, mesmo sem isolamento térmico, permitem que sejam aferidas temperaturas por volta de $90^{\circ} \mathrm{C}$ na entrada da linha de montagem com oscilações que chegam a uma média de $80^{\circ} \mathrm{C}$.

Nas preparações à base de proteína animal, constatou-se que as temperaturas de cocção variam entre $94^{\circ} \mathrm{C}$ a $99^{\circ} \mathrm{C}$, quando em manutenção varia entre $64^{\circ} \mathrm{C}$ e $92^{\circ} \mathrm{C}$, variação causada pelo tipo de conservação, se em carros cubas rasas ou fundos direto na linha de produção sem fonte de calor ou isolamento térmico.

As guarnições, farofa de farinha de mandioca ou de milho, embora atinjam durante a cocção uma média de $77^{\circ} \mathrm{C}$ e $100^{\circ} \mathrm{C}$, respectivamente, permanecem na área de envase com uma temperatura média de $60^{\circ} \mathrm{C}$ durante a distribuição.

As saladas cozidas ofertadas apresentaram temperatura de cocção média de $95^{\circ} \mathrm{C}$ e de manutenção/envase entre $64^{\circ} \mathrm{C}$ e $69^{\circ} \mathrm{C}$.

Do Nascimento (2017), ao comparar os achados com as legislações RDC n²16/2014 e CVS n 5/2013 apresentou 100\% de inadequação em relação as guarnições, já as saladas cozidas apresentaram 55,6\% de inconformidades relacionadas as temperaturas, valores estes distantes dos achados no presente trabalho.

Relacionando-se o tempo de envase, transporte e distribuição observou-se que o tempo total sofre uma variação entre 02 horas e 03 horas entre o início do envase e o final da distribuição. Os resultados finais não implicam ou comprometem a qualidade das refeições, já que o tempo de exposição abaixo de $60^{\circ} \mathrm{C}$ não ultrapassa o máximo de uma hora.

Ao analisar o processo como um todo, começando com a cocção e seguindo com o envase, transporte e distribuição no local pode-se observar que não excede o tempo máximo de 6 horas do final da cocção até o recebimento (Figura 1). 
Figura 1: Demonstrativo da hora média do processo produtivo.

Tempo total médio: $04 \mathrm{~h} 24 \mathrm{~min}$

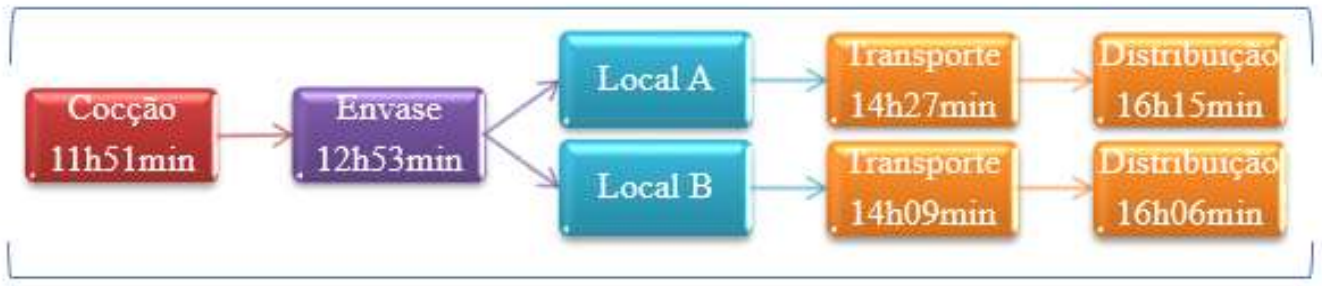

Tempo total médio: $04 \mathrm{~h} 15 \mathrm{~min}$

Fonte: Autores.

Ao tempo médio apresentado na Figura 1 pode-se ainda adicionar 50 minutos, que é o tempo médio que a unidade necessita, ato do recebimento, para realizar a distribuição das refeições a todos os comensais. Somando esse tempo a unidade A teremos um tempo médio de 5 horas e 14 minutos e para unidade B 5 horas e 5 minutos.

Na Tabela 3 pode ser observado que nenhuma quentinha foi expedida abaixo de $60^{\circ} \mathrm{C}$, o que reduziria o prazo da garantia da alimentação ofertada, além disso, as quentinhas que são transportadas em caixas térmicas tampadas com capacidade máxima para 24 unidades favorecem a conservação da temperatura pelo efeito estufa presente no meio.

Tabela 3: Representação numérica e percentual das temperaturas das preparações na etapa de transporte. Fortaleza, 2019.

\begin{tabular}{l|r|r|r|r|r|r|r|r|r}
\hline & $\begin{array}{c}\text { Abaixo } \\
\text { de } \mathbf{6 0}^{\circ} \mathbf{C}\end{array}$ & $\%$ & $\begin{array}{c}\text { Entre } \\
\mathbf{6 0}^{\circ} \mathbf{e}\end{array}$ & $\boldsymbol{\%}$ & $\begin{array}{c}\text { Entre } \\
\mathbf{6 5}^{\circ} \mathbf{C}\end{array}$ & $\boldsymbol{\%}$ & $\begin{array}{c}\text { Acima } \\
\mathbf{d e} \\
\mathbf{7 0}^{\circ} \mathbf{C}\end{array}$ & $\begin{array}{c}\text { \% } \\
\mathbf{7 0}^{\circ} \mathbf{C}\end{array}$ & $\begin{array}{c}\text { Total } \\
\text { Amostras } \\
\text { (Dias) }\end{array}$ \\
\hline Local A & 0 & $0,0 \%$ & 4 & $50,0 \%$ & 2 & $25,0 \%$ & 2 & $25,0 \%$ & 8 \\
\hline Local B & 0 & $0,0 \%$ & 3 & $37,5 \%$ & 2 & $25,0 \%$ & 3 & $37,5 \%$ & 8 \\
\hline
\end{tabular}

Fonte: Dados obtidos pelo pesquisador.

O estudo realizado por Moura Júnior (2018) encontrou resultados semelhantes, onde a maioria das refeições envasadas avaliadas apresentaram temperatura final acima de $60^{\circ} \mathrm{C}$, sendo um total de 383 amostras $(95,75 \%)$ e abaixo de $60^{\circ} \mathrm{C}, 17$ amostras (4,25\%). Achados semelhantes foram encontrados por Gonzaga, Lira e Fonseca (2021), onde todos gêneros alimentícios, com exceção aqueles que são servidos frios, apresentaram temperatura de recebimento acima de $60^{\circ} \mathrm{C}$ como preconizado pela legislação.

Analisando-se os dados da pesquisa, observa-se que a maioria das refeições envasadas avaliadas no estudo apresentou temperatura final igual ou acima do mínimo definido e/ou recomendado para o consumo durante o recebimento e distribuição, resultado em valores acima de $60^{\circ} \mathrm{C}$, em um total de 371 amostras $(92,7 \%)$. Além disso, vale ressaltar que as 29 amostras que se encontravam abaixo da temperatura limite $(7,3 \%)$ foram distribuídas em tempo inferior a uma hora.

\section{Conclusão}

O binômio tempo e temperatura de refeições transportadas para o jantar de instituições de privação de liberdade na Região Metropolitana de Fortaleza-CE encontra-se dentro dos parâmetros previstos na legislação sanitária vigente e literatura relacionada.

Desta forma, torna-se evidente que os resultados dão credibilidade ao processo produtivo e seu produto final, refeição pronta e envasada, contribuindo para o controle de qualidade e a produção de alimentos seguros transportados. 
Contudo, são necessários mais estudos sobre a alimentação carcerária em geral, devido à escassez de conteúdo científico em relação a este tema.

\section{Referências}

Abreu, E. S., Simony, R. F., da Silva, A. P., Kuribayashi, C. L., da Silva, J. C., \& de Vito, P. S. (2012). Monitoramento da temperatura de refeições quentes transportadas porcionadas. e-Scientia, 5(1), 03-08.

Associação Brasileira das Empresas de Refeições Coletivas. Manual ABERC de Práticas de Elaboração e Serviço de Refeições para Coletividades 2015. (11a ed.), ABERC, 2015.

Audi, C. A. F., Santiago, S. M., Andrade, M. G. G., Assumpção, D., Francisco, P. M. S. B., Segall-Corrêa, A. M., \& Pérez-Escamilla, R. (2018). Ultra-processed foods consumption among inmates in a women's prison in São Paulo, Brazil. Revista espanola de sanidad penitenciaria, $20(3), 87$.

Borges, N. R., Moura, B. A., Vieira, C. F. S., Santos, D. D. M., Almeida, L. J., \& Zuniga, A. D. G. (2016). Avaliação do Binômio Tempo-Temperatura das refeições de um restaurante na cidade de Palmas-TO. DESAFIOS-Revista Interdisciplinar Da Universidade Federal Do Tocantins, 3(2), 90-98.

Bozatski, L. C., Moura, P. N. D., \& Novello, D. (2011). Análise do binômio tempo x temperatura na distribuição de alimentos em unidades de alimentação e nutrição comerciais do município de Guarapuava, Paraná. Revista Higiene Alimentar, 76(181), 10-15.

Brasil (2009). Congresso Nacional. Câmara dos Deputados. Comissão Parlamentar de Inquérito do Sistema Carcerário. CPI sistema carcerário

Brasil. (2004). Ministério da Saúde. Agência Nacional de Vigilância Sanitária (ANVISA). Resolução da Diretoria Colegiada (RDC) ${ }^{\circ} 216$, de 15 de setembro de 2004. Estabelece procedimentos de Boas Práticas para serviço de alimentação, garantindo as condições higiênico-sanitárias do alimento preparado. Diário Oficial da União. https://bvsms.saude.gov.br/bvs/saudelegis/anvisa/2004/res0216_15_09_2004.html

Cardoso, F. K. P., \& Tarzia, A. (2016). Análise das Temperaturas dos Alimentos Servidos em um Restaurante Universitário da Cidade De Curitiba/PR. Revista Eletrônica Biociências, Biotecnologia e Saúde, 7(16), 76-85.

Chaves, J. B. P. (2011). Boas práticas de fabricação (BPF) para restaurantes, lanchonetes e outros serviços de alimentação. In Boas práticas de fabricação (BPF) para restaurantes, lanchonetes e outros serviços de alimentação (pp. 68-68).

Chouman, K., Pponsano, E. H. G., \& Michelin, A. D. F. (2010). Qualidade microbiológica de alimentos servidos em restaurantes self-service. Revista do Instituto Adolfo Lutz (Impresso), 69(2), 261-266.

Dariva, R., \& Alberto, O. H. (2013). Atuação do nutricionista líder em unidade de alimentação e nutrição no segmento de refeições transportadas para penitenciárias em Curitiba-PR e Região Metropolitana. Administração de Empresas em Revista, 1(8), 72-93.

Figueiredo, R. M. As armadilhas de Uma Cozinha. Coleção Higiene dos Alimentos Manole. Manole (3a ed.).

Gatherer, A. (2007). Health in prisons: a WHO guide to the essentials in prison health. WHO Regional Office Europe.

Guerra, A.F. (2016). Fatores do crescimento microbiano. Valença.

Gonzaga, S. O., Lira, C. R. N. de, \& Fonseca, M. da C. P. da. (2021). Qualidade de refeições transportadas em restaurante universitário. Research, Society and Development, 10(8), e34110817430. https://doi.org/10.33448/rsd-v10i8.17430

Kawasaki, V. M., Cyrillo, D. C., \& Machado, F. M. S. (2007). Custo-efetividade da produção de refeições coletivas sob o aspecto higiênico-sanitário em sistemas cook-chill e tradicional. Revista de nutrição, 20, 129-138.

Moura Júnior, F. B. (2018). Controle Microbiológico e de temperatura de refeições transportadas para unidades prisionais. Dissertação. Fortaleza.

Nascimento, L. A. (2017). Binômio tempo e temperatura de preparações em serviço de alimentação transportada.

Paredes, L. C. (2005). Avaliação da Alimentação Terceirizada no Sistema Penitenciário do Paraná. Monografia. Curitiba.

Peixoto, T., Carneiro, A. C. L. L., \& Cardoso, L. (2020). Análise do binômio tempo e temperatura de preparações à espera para distribuição em um restaurante universitário. Revista Da Associação Brasileira De Nutrição-RASBRAN, 11(1), 142-161.

Ricarte, M. P. R., Fé, M. A. B. M., da Silva Santos, I. H. V., \& Lopes, A. K. M. (2021). Avaliação do desperdício de alimentos em uma unidade de alimentação e nutrição institucional em Fortaleza-CE. Saber Científico (1982-792X), 1(1), 158-175.

Santos, V. N., \& Bassi, S. M. (2015). Avaliação da temperatura dos equipamentos e alimentos servidos em unidades de alimentação e nutrição na cidade de São Paulo. Revista Científica Linkania Master, 5(1).

São Paulo. Centro de Vigilância Sanitária. Portaria CVS 5/2013, de 09 de abril de 2013. Centro de Vigilância Sanitária, da Coordenadoria de Controle de Doenças, da Secretaria de Estado da Saúde de São Paulo, São Paulo.

Silva Júnior, E. A. (2014). Manual de Controle Higiênico Sanitário em Serviços de Alimentação (7a ed.), Varela.

Tondo, E. C. \& Bartz, S. Microbiologia e Sistemas de Gestão da Segurança de Alimentos. 2. Editora Sulina. 